\title{
La propriété de Kneser globale et le problème aux limites
}

\author{
Par \\ Masuo Hukuhara
}

\section{Introduction}

M. Nagumo [2] a obtenu de beaux résultats concernant le problème aux limites pour 1'équation différentielle ordinaire du second ordre

$$
d^{2} x / d t^{2}=f(t, x, d x / d t)
$$

Le problème s'énonce: chercher des solutions prenant des valeurs $\beta, \beta^{\prime}$ pour $t=\alpha, \alpha^{\prime}$, les valeurs $\alpha, \alpha^{\prime}$ et $\beta, \beta^{\prime}$ étant données d'avance. De diverses améliorations ont été apportées par lui-même et par le regretté H. Okamura.

Le problème se généralise au cas de 1'équation différentielle ordinaire du $n^{\text {ième }}$ ordre:

$$
d^{n} x / d t^{n}=f\left(t, x, d x / d t, \cdots, d^{n-1} x / d t^{n-1}\right),
$$

comme il suit: chercher des solutions prenant des valeurs $\beta_{1}, \cdots, \beta_{n}$ pour $t=\alpha_{1}, \cdots, \alpha_{n}$, les valeurs $\alpha_{1}, \cdots, \alpha_{n}$ et $\beta_{1}, \cdots, \beta_{n}$ étant données d'avance.

Appelons-le problème I. D'autre part, concernant un système d'équations différentielles ordinaires

$$
d x_{i} / d t=f_{i}\left(t, x_{1}, \cdots, x_{n}\right), \quad i=1, \cdots, n,
$$

nous pouvons nous proposer le problème: chercher des solutions satisfaisant à la condition

$$
x_{i}\left(\alpha_{i}\right)=\beta_{i} \quad i=1, \cdots, n,
$$

les valeurs $\alpha_{1}, \cdots, \alpha_{n}$ et $\beta_{1}, \cdots, \beta_{n}$ étant données d'avance.

Appelons-le problème II. Nous avons appliqué des théorèmes 
d'existence pour ce problème à l'étude des allures des singularités que présente la solution d'un système différentiel ordinaire.

Les deux problèmes sont intimement liés de sorte que l'on peut transformer le problème I au problème II par un changement de variables simple. Nous l'avons remarqué dans mon livre [9], et $\mathrm{M}$. Nagumo a achevé le calcul et a obtenu le transformé sous une forme élégante [5].

Concernant le problème II, nous avons obtenu, dans le cas de $n=2$, un théorème d'existence de nature assez générale [5]. Depuis lors j'avais une conjecture sur la possibilité d'obtenir un théorème d'existence dont découlent comme cas particuliers les résultats cités plus haut. Nous voulons montrer, dans le présent article, que l'on peut répondre affirmativement à notre conjecture si l'on remplace les inégalités non strictes par les inégalités strictes dans les propositions de M. Nagumo et de H. Okamura. La condition d'existence ne dépendra, comme on le verra, que du comportement du second membre de l'équation sur la frontière du domaine où il est défini. Si l'on veut étendre notre résultat au cas de $n$ quelconque, on devrait d'approfondir encore la propriété de Kneser.

\section{耳. Famille de caractéristiques}

\section{Hypothèses.}

Considérons une famille $\mathbb{F}$ de fonctions à valeurs dans $R^{n}$ satisfaisant aux hypothèses suivantes.

1.1. Chaque fonction de la famille $\mathbb{F}$ ainsi que son graphique est appelée caractéristique. Elle est définie et continue dans un intervalle compact. Il peut arriver qu'elle n'est définie qu'en un seul point.

1. 2. Si deux caractéristiques $x$ et $y$ se rencontrent en un point d'abscisse $t=\alpha$, la fonction $z$ qui coincide avec $x$ pour $t \leqq \alpha$ et avec $y$ pour $t \geqq \alpha$ est aussi une caractéristique. 
1. 3. La famille $\boldsymbol{F}$ est compacte dans l'espace distancié( ${ }^{(1)} \boldsymbol{D}$ dont les éléments sont des compacts dans $R^{n+1}$. L'ensemble des caractéristiques remplit donc un ensemble compact $\mathfrak{D}$ dans $R^{n+1}$.

1.4. Chaque caractéristique est maximale, c'est-à-dire elle n'est plus prolongeable vers gauche ni vers droite. L'extrémité gauche ne se trouve pas à l'intérieur de $\mathfrak{D}$. Elle est appelée point extrême gauche de $\mathfrak{D}$. On définit de même point extrême droit. Il est supposé situé à la frontière de $\mathfrak{D}$. Un point extrême gauche peut être en même temps un point extrême droit, auquel cas la caractéristique correspondante ne contient que ce point extrême.

1.5. Une caractéristique se partage par un de ses points en deux parties que nous appellerons demi-caractéristique gauche et demicaractéristique droite. Une demi-caractéristique gauche peut se réduire à un point extrême gauche. Il en est de même de demicaractéristique droite. $A$ désignant un point de $\mathfrak{D}$, l'ensemble des demi-caractéristiques droites issues de $A$ remplit un ensemble que nous désignons par $3^{+}(A)$ et appelons zone d'émission de $A$ à droite. Si un point $A \in \mathscr{D}$ d'abscisse $\alpha$ n'est pas extrême à droite, les demi-caractéristiques droites issues de $A$ existent dans un intervalle assez petit $[\alpha, \tau]$ et la section de $3^{+}(A)$ par l'hyperplan $t=\tau$ est un continu. Un point ayant cette propriété est appelé point kneserien à droite. On peut donc dire qu'un point de $\mathfrak{D}$ non extrême à droite est kneserien à droite.

On définit de même zone d'émission à gauche $3^{-}(A)$ et point kneserien à gauche.

La famille satisfaisant à cette hypothèse est appelée famille kneserienne.

2. Compacité.

(5) désignant une partie compacte de $\mathfrak{D}$, la zone d'émission de $\mathfrak{F}$

(1) La distance $\rho(A, B)$ entre deux compacts $A$ et $B$ est définie par $\rho(A, B)=\inf \left\{\delta ; U_{0}(A) \supset B, U_{\delta}(B) \supset A\right\}$,

où $U_{\delta}(A)$, par exemple, désigne l'ensemble des points distants de $A$ moins de $\delta$. 
à droite $3^{+}(\mathfrak{F})$ est définie par

$$
B^{+}(\mathfrak{F})=\bigcup\left\{Z^{+}(P) ; P \in \mathfrak{F}\right\} \text { 。 }
$$

Si $P$ est un point extrême à droite, $\Im^{+}(P)$ ne contient que $P$.

On définit de même zone d'émission de (F à gauche $3^{-}$(F). La réunion de $3^{ \pm}(\mathfrak{F})$ sera appelée zone d'émission de $\mathfrak{F}$ et sera désignée par $3(\xi)$.

Désignons par $\mathfrak{F}^{+}(\mathfrak{F})$ la famille des demi-caractéristiques droites issues de $\mathfrak{F}$, par $\mathfrak{F}^{-}(\mathfrak{F})$ celle des demi-caractéristiques gauches issues de $\mathfrak{F}$ et par $\mathfrak{F}(\mathfrak{F})$ celle des caractéristiques qui recontrent $\mathfrak{F}$.

Proposition 1. Les familles $\mathfrak{F}^{ \pm}(\mathfrak{F})$ et $\mathfrak{F}(\mathfrak{F})$ sont des ensembles compacts dans l'espace $\mathbf{D}$.

Considérons une suite $\left\{x_{k}^{\prime}\right\}$ extraite de $\mathfrak{F}^{+}(\mathfrak{F}) . \quad x_{k}^{\prime}$ est une partie droite d'une caractéristique $x_{k}$. On peut extraire de la suite $\left\{x_{k}\right\}$ une suite partielle convergente dans $\mathbb{D}$, laquelle nous désignerons par $\left\{y_{k}\right\}$. La suite partielle correspondante de $\left\{x_{k}^{\prime}\right\}$ sera désignée par $\left\{y_{k}^{\prime}\right\}$. Si $A_{k}$ et $B_{k}$ représentent respectivement les extrémités gauches de $x_{k}^{\prime}$ et $y_{k}^{\prime},\left\{B_{k}\right\}$ est une suite partielle de la suite $\left\{A_{k}\right\}$. Celle-ci étant extraite d'un compact $\left[F\right.$, on peut supposer la suite $\left\{B_{k}\right\}$ convergente sans perdre la généralité. La caractéristique limite $y$ de la suite $\left\{y_{k}\right\}$ passe par le point limite $B$ de la suite $\left\{B_{k}\right\}$. \& étant compact, $B$ appartient à $\left\{\right.$, et la suite $\left\{y_{k}^{\prime}\right\}$ converge vers la demi-caractéristique $y^{\prime}$ qui est la partie droite de $y$ limitée par $B$. $\mathfrak{F}^{+}(\mathfrak{F})$ est donc compacte dans $\mathbb{D}$.

On démontre de même la compacité de $\mathfrak{F}^{-}(\mathfrak{F})$. La démonstration de la compacité de $\mathfrak{F}(\Im)$ est plus facile.

Puisque l'ensemble rempli par les caractéristiques d'une famille compacte dans $\mathbb{D}$ est un compact dans $R^{n+1}$, on a la

Proposition 2. Les ensembles $3^{ \pm}(\mathfrak{F})$ et $3($ (5) sont compacts.

\section{Propriété de Kneser globale.}

Une partie compacte $\sqrt{5}$ de $3^{+}(\mathfrak{F})$ sera appelée section transversale de $3^{+}(\mathbb{5})$ si la condition suivante est remplie:

Chaque demi-caractéristique de $\mathfrak{F}^{+}(\mathfrak{F})$ rencontre $\mathfrak{F}$ en un point et 
en un seul.

On a alors la

Proposition 3. Si [ est un continu dans D et si (5) est une section transversale de $3^{+}(5)$, 5 est aussi un continu.

Supposons le contraire. (5 est alors la réunion de deux compacts disjoints $\mathfrak{F}_{1}$ et $\mathfrak{F}_{2}$. La famille $\mathfrak{F}^{+}(\mathfrak{F})$ se partage en deux familles disjointes: la famille $\mathfrak{F}_{1}$ formée des demi-caractéristiques de $\mathfrak{F}^{+}(\mathfrak{F})$ rencontrant $\mathfrak{F}$ en un point de $\mathfrak{F}_{1}$, et la famille $\mathfrak{F}_{2}$ formée des demicaractéristiques de $\mathfrak{F}^{+}(\mathfrak{F})$ rencontrant $\mathfrak{F}$ en un point de $\mathfrak{F}_{2}$. $\mathfrak{F}_{1}$ et $\mathfrak{F}_{2}$ sont évidemment compactes dans et remplissent des parties compactes $\mathfrak{F}_{1}$ et $\mathfrak{F}_{2}$ de $3^{+}(\mathfrak{F})$, et la réunion $\mathfrak{F}_{1} \cup \mathfrak{F}_{2}$ coïncide avec $3^{+}(\mathfrak{F})$. Celle-ci étant un continu, $\mathfrak{F}_{1} \cap \mathfrak{F}_{2}$ n'est pas vide. Soit $A$ un point de $\mathfrak{F}_{1} \Gamma_{1} \mathfrak{F}_{2}$ ayant la plus grande abscisse $\alpha$. D'après la propriété de Kneser locale, la section $\subseteq$ de $3^{+}(A)$ par l'hyperplan $t=\tau$ est un continu pourvu que $\tau-\alpha>0$ soit assez petit. D'après la propriété I. 2, $\mathfrak{S}$ est contenu dans $3^{+}(\mathfrak{F})$. Par suite $\mathfrak{S} \cap \mathfrak{F}_{1} \cap \mathfrak{F}_{2}$ ne serait pas vide. $\mathfrak{F}_{1} \cap \mathfrak{F}_{2}$ contiendrait donc un point d'abscisse plus grande que $\alpha$, contrairement à la définition de $a$.

On a évidemment une proposition analogue pour $\mathfrak{F}^{-}(\mathfrak{F})$.

Section transversale de $3(\mathbb{F})$ est une partie compacte (5) de 3 (F) satisfaisant à la condition suivante:

Chaque caractéristique de $\mathfrak{F}(\mathfrak{F})$ rencontre $\mathfrak{F}$ en un point et en un seul.

Proposision 4. Si [5 est une partie continue de (D) et si (5) est une section transversale de $3(\sqrt{5})$, (5 est un continu.

Supposons le contraire. (5 est alors la réunion de deux compacts disjoints $\mathfrak{r}_{1}$ et $\mathfrak{r}_{2}$. La famille $\mathfrak{F}(\mathfrak{F})$ se partage en deux familles compactes disjointes: la famille $\mathfrak{J}_{1}$ formée des caractéristiques de $\mathfrak{F}(\mathfrak{F})$ rencontrant $\mathfrak{F}$ en un point de $\mathfrak{F}_{1}$ et la famille $\mathfrak{F}_{2}$ formée des caractéristiques de $\mathfrak{F}(\mathfrak{F})$ rencontrant $\mathfrak{F}$ en un point de $\mathfrak{F}_{2}$. $\mathfrak{F}_{1}$ et $\mathfrak{F}_{2}$ remplissent des parties compactes $\xi_{1}$ et $\xi_{2}$ de $3(\xi)$, et la réunion $\mathfrak{F}_{1} \cup \mathfrak{F}_{2}$ coïncide avec $3(\mathfrak{F})$. Donc, l'intersection $\mathfrak{F}_{\mathfrak{F}} \cap \mathfrak{F}_{1} \cap \mathfrak{F}_{2}$ n'est pas 
vide. Si $A$ appartient à $\mathfrak{F} \cap \mathfrak{F}_{1} \cap \mathfrak{F}_{2}$, on a

$$
3(A) \cap \mathfrak{\mho}_{i} \neq 0, \quad i=1,2 .
$$

L'intersection

$$
\mathfrak{\Im}^{\prime}=3(A) \cap \Subset
$$

est la réunion de deux compacts disjoints $\mathfrak{S}^{\prime} \cap \mathfrak{\mho}_{i}, i=1,2$. Elle est évidemment une intersection transversale de $3(A)$. Par suite elle est contenue dans l'un des ensembles $3^{ \pm}(A)$, car si elle contenait un point $B$ de $3^{-}(A)$ et un point $C$ de $3^{+}(A)$, il existeraient une caractéristique $y \in \mathfrak{F}^{-}(A)$ rencontrant $\mathfrak{S}^{\prime}$ en $B$ et une caractéristique $z \in \mathfrak{F}^{+}(A)$ rencontrant $\mathfrak{S}^{\prime}$ en $C$. Alors la caractéristique $x$ qui coïncide avec $y$ à gauche de $A$ et avec $z$ à droite de $A$ appartiendrait à $\mathfrak{F}(A)$ et rencontrerait $\mathfrak{S}^{\prime}$ en deux points $B$ et $C$, et $\mathfrak{C}^{\prime}$ ne pourrait être une intersection transversale de $3(A)$.

La proposition 3 montre ensuite que $\widetilde{C}^{\prime}$ est un continu. C'est absurde, parce que $\mathfrak{C}^{\prime}$ était la réunion de deux compacts disjoints.

\section{Problème aux limites.}

Appelons frontière droite l'ensemble des points extrêmes à droite et frontière gauche l'ensemble des points extrêmes à gauche.

Proposition 5. La frontière droite $\mathfrak{B}^{+}$et la frontière gauche $\mathfrak{B}^{-}$sont des ensembles compacts.

Supposons en effet qu'une suite $\left\{A_{k}\right\}$ extraite de $\mathfrak{B}^{+}$converge vers un point $A$. Soit $x_{k}$ une caractéristique dont l'extrémité droite est $A_{k}$. On peut extraire de la suite $\left\{x_{k}\right\}$ une suite partielle $\left\{y_{k}\right\}$ convergeant vers une caractéristique $y$. L'extrémité droite de $y$ est nécessairement $A$. $A$ appartient donc à $\mathfrak{B}^{+}$.

Appelons point latéral un point frontière de $\mathfrak{D}$ qui n'est pas un point extrême à gauche ni un point extrême à droite. Par suite par un point latéral passe au moins une caractéristique.

Théorème d'existence 1. Supposons qu'il n'y ait aucun point latéral. Soient $\&$ et ${ }^{\prime}$ des continus contenus respectivement dans $\mathfrak{R}^{-}$et $\mathfrak{B}^{+}$, et satisfaisant à la condition suivante: 
(5) contient des points de $\mathfrak{B}^{+}$et il n'y a aucune partie continue de $\mathfrak{B}^{+}$contenant $\mathfrak{F}^{\prime} \cap \mathfrak{B}^{+}$et disjoint de $\mathfrak{F}^{\prime}$.

Il existe alors au moins une caractéristique dont les extrémités se trouvent dans (5) et [5'.

En effet, l'intersection $\sqrt{5}$ de $Z^{+}(\mathfrak{S})$ et $\mathfrak{B}^{+}$étant évidemment une intersection transversale de $3^{+}(\mathfrak{S})$, elle est un continu. Elle contient $\mathfrak{G} \cap \mathfrak{B}^{+}$. Elle ne peut contenir aucun point de $\mathfrak{B}^{-}$sauf ceux de $\mathfrak{G} \cap \mathfrak{B}^{+}$. Elle contient donc au moins un point de $\breve{F}^{\prime}$. Si $C$ est un point d'intersection de $\mathfrak{F}^{\prime}$ et $Z^{+}(\mathfrak{F})$, il existe au moins une caractéristique de $Z^{+}(\mathfrak{s})$ qui joint $\sqrt{5}$ à $C$. Cette caractéristique répond à la question.

Note. Pendant la conversation avec Prof. Shimada, il a indiqué que pour obtenir la conclusion du théorème, il suffit de montrer que la section $\sqrt{ }$ ( est un continu. J'avais déjà obtenu la propriété de Kneser globale, en introduisant la notion de section transversale, et son indication m'a conduit immédiatement à la démonstration ci-dessus.

\section{Equation différentielle}

5. Equation différentielle ordinaire.

Considérons l'équation différentielle ordinaire

$$
d x / d t=f(t, x)
$$

où $f$ est une fonction à valeurs dans $R^{n}$ définie et continue dans un compact $\mathfrak{D} \subset R^{n+1}, x$ désignant point variable dans $R^{n}$. Appelons caractéristique toute solution qui n'est plus prolongeable vers gauche ni vers droite. Alors les extrémités d'une caractéristique se trouvent sur la frontière $\mathfrak{B}$ de $\mathfrak{D}$.

Nous dirons que le vecteur $(1, f(\alpha, a))$ lié en $A$ se dirige vers l'extérieur (l'intérieur) de $\mathfrak{D}$, si l'on a condition suivante:

Tout point $(t, x)$ tel que

$$
0<t-\alpha \leqq \varepsilon, \quad|x-a-(t-\alpha) f(\alpha, a)| \leqq \varepsilon(t-\alpha)
$$


est extérieur (intérieur) à $\mathfrak{D}$ pourvu que $\varepsilon>0$ soit assez petit.

Si l'on a cette condition où l'on a remplacé $t-\alpha$ par $\alpha-t$, nous dirons que le vecteur $-(1, f(\alpha, a))$ lié en $A$ se dirige vers l'extérieur (l'intérieur) de $\mathfrak{D}$.

Proposition 6. L'ensemble des caractéristiques de l'équation différentielle (5.1) remplit les hypothèses I. $1 \sim 5$, si l'on a la condition suivante:

L'ensemble des points frontières $(t, x)$ où le vecteur $(1, f(t, x))$ se dirige vers l'extérieur de $\mathfrak{D}$ est fermé ainsi que celui des points frontières $(t, x)$ où le vecteur $-(1, f(t, x))$ se dirige vers l'extérieur de $\mathfrak{D}$; si l'un des vecteurs $\pm(1, f(t, x))$ ne se dirige pas vers l'extérieur de $\mathfrak{D}$, il se dirige vers l'intérieur de $\mathfrak{D}$, et l'autre se dirige vers l'extérieur de $\mathfrak{D}$.

On voit immédiatement que toutes les hypothèses sont remplies sauf la compacité. Nous voulons donc démontrer celle-ci seulement.

Considérons une suite de caractéristiques $\left\{x_{k}\right\}$, et désignons par $\left(\alpha_{k}, a_{k}\right)$ et $\left(\alpha_{k}^{\prime}, a_{k}^{\prime}\right)$ l'extrémité gauche et l'extrémité droite de $x_{k}$. On peut extraire de la suite $\left\{x_{k}\right\}$ une suite partielle telle que les suites partielles correspondantes $\left\{\left(\beta_{k}, b_{k}\right)\right\}$ et $\left\{\left(\beta_{k}^{\prime}, b_{k}^{\prime}\right)\right\}$ des suites $\left\{\left(\alpha_{k}, a_{k}\right)\right\}$ et $\left\{\left(\alpha_{k}^{\prime}, a_{k}^{\prime}\right)\right\}$ soient convergentes. Si $(\beta, b)$ et $\left(\beta^{\prime}, b^{\prime}\right)$ sont les points limites respectifs des suites $\left\{\left(\beta_{k}, b_{k}\right)\right\}$ et $\left\{\left(\beta_{k}^{\prime}, b_{k}^{\prime}\right)\right\}$, on peut supposer d'après l'équi-continuité des caractéristiques, que la suite $\left\{y_{k}\right\}$ converge vers une caractéristique $y$ uniformément à l'intérieur de l'intervalle $\left[\alpha, \alpha^{\prime}\right]$. L'hypothèse de la proposition implique que le vecteur $-(\alpha$, $f(\alpha, a))$ lié en $(\alpha, a)$ et le vecteur $\left(\alpha^{\prime}, f\left(\alpha^{\prime}, a^{\prime}\right)\right)$ lié en $\left(\alpha^{\prime}, a^{\prime}\right)$ se dirigent vers l'extérieur de $\mathfrak{D}$ et les points $(\alpha, a)$ et $\left(\alpha^{\prime}, a^{\prime}\right)$ sont les extrémités de la caractéristique $y$. Grâce à l'équi-continuité des caractéristiques, la suite $\left\{y_{k}\right\}$ converge vers $y$ dans $\boldsymbol{D}$, d'où résulte la compacité.

\section{Hypothèses sur l'équation différentielle.}

Supposons les hypothèses suivantes remplies.

II. 1. $f$ est définie et continues dans une partie compacte $\mathfrak{D}$ de 

$R^{n+1}$

II. 2. L'ensemble $\mathfrak{B}^{+}$des points frontières de $\mathfrak{D}$ où le vecteur $(1, f(t, x))$ se dirige vers l'extérieur de $\mathfrak{T}$ est fermé, ainsi que l'ensemble $\mathfrak{B}^{-}$des points frontières de $\mathfrak{D}$ où le vecteur $-(1, f(t, x))$ se dirige vers l'extérieur de $\mathfrak{D}$.

II. 3. Si l'un des vecteurs $\pm(1, f(t, x))$ liés en un point frontière $(t, x)$ ne se dirige pas vers l'extérieur de $\mathfrak{D}$, il se dirige vers l'intérieur de $\mathfrak{D}$, tandis que l'autre se dirige vers l'extérieur de $\mathfrak{D}$.

II. 4. $\mathfrak{S}$ et $\mathfrak{F}^{\prime}$ sont respectivement des parties continues de $\mathfrak{B}^{-}$ et $\mathfrak{B}^{+}$, l'intersection $\mathfrak{S} \cap \mathfrak{B}^{+}$n'est pas vide, et il n'y a aucune partie continue du second contenant $\mathfrak{S} \cap \mathfrak{B}^{+}$et disjoint de $\mathfrak{S}^{\prime}$.

Dans ces hypothèses nous pouvons appliquer le théorème d'existence 1 et obtenons le

Théorème d'existence 2. Il existe au moins une caractéristique qui joint $\sqrt{5}$ et $\mathfrak{S}^{\prime}$.

\section{Rappel des résultats de M. Nagumo.}

Considérons 1'équation différentielle ordinaire du second ordre

$$
x^{\prime \prime}=f\left(t, x, x^{\prime}\right),
$$

où l'inconnue $x$ est une fonction réelle d'une variable réelle $t, x^{\prime}, x^{\prime \prime}$ désignant ses dérivées première et seconde respectivement. $f$ est supposée définie et continue dans le domaine

$$
\alpha \leqq t \leqq \alpha^{\prime}, \quad \underline{\omega}(t) \leqq x \leqq \bar{\omega}(t), \quad\left|x^{\prime}\right|<\infty,
$$

où $\omega$ et $\overline{\boldsymbol{\omega}}$ sont des fonctions deux fois dérivables dans l'intervalle $\left[\alpha, \alpha^{\prime}\right]$. Supposons de plus que l'on ait

$$
\begin{aligned}
& \omega^{\prime \prime}(t)>f\left(t, \underline{\omega}(t), \omega^{\prime}(t)\right), \\
& \bar{\omega}^{\prime \prime}(t)<f\left(t, \bar{\omega}(t), \bar{\omega}^{\prime}(t)\right)
\end{aligned}
$$

et

$$
\left|f\left(t, x, x^{\prime}\right)\right| \leqq \varphi\left(\left|x^{\prime}\right|\right)
$$


$\varphi$ désignant une fonction continue et positive, telle que

$$
\int_{0}^{\infty} \frac{u d u}{\varphi(u)}=+\infty .
$$

$\mathrm{Si}$

$$
\underline{\omega}(\alpha)=\beta=\bar{\omega}(\alpha), \quad \underline{\omega}\left(\alpha^{\prime}\right) \leqq \beta^{\prime} \leqq \bar{\omega}\left(\alpha^{\prime}\right),
$$

il existe au moins une solution qui prend la valeur $\beta$ en $t=\alpha$ et la valeur $\beta^{\prime}$ en $t=\alpha^{\prime}$.

C'est un théorème d'existence établi par M. Nagumo en 1937 [2]. Il l'a précisé [6], en remplaçant les inégalités strictes (7.3) par les inégalités non strictes

$$
\begin{aligned}
& \underline{\omega}^{\prime \prime}(t) \geqq f\left(t, \underline{\omega}(t), \underline{\omega}^{\prime}(t)\right), \\
& \overline{\bar{\omega}}^{\prime \prime}(t) \leqq f\left(t, \overline{\boldsymbol{\omega}}(t), \overline{\bar{\omega}}^{\prime}(t)\right)
\end{aligned}
$$

et le domaine non borné (7.2) par le domaine borné

$$
\alpha \leqq t \leqq \alpha^{\prime}, \quad \underline{\omega}(t) \leqq x \leqq \bar{\omega}(t), \quad \underline{\Omega}(t, x) \leqq x^{\prime} \leqq \bar{\Omega}(t, x),
$$

où $\underline{\Omega}$ et $\bar{\Omega}$ sont des fonctions continues admettant les dérivées continues $\underline{\Omega}_{t}, \cdots, \overline{\Omega_{x}}$ et satisfaisant aux inégalités

$$
\underline{\Omega}(t, \underline{\bar{\omega}}(t)) \leqq \underline{\bar{\omega}}^{\prime}(t) \leqq \bar{\Omega}(t, \underline{\bar{\omega}}(t))
$$

et

$$
\begin{aligned}
& f(t, x, \underline{\underline{Q}}(t, x))-\underline{\underline{\Omega}}_{t}(t, x)-\underline{\Omega}_{x}(t, x) \underline{\Omega}(t, x)>0, \\
& f(t, x, \bar{\Omega}(t, x))-\bar{\Omega}_{t}(t, x)-\bar{\Omega}_{x}(t, x) \bar{\Omega}(t, x)<0
\end{aligned}
$$

pour

$$
\alpha \leqq t \leqq \alpha^{\prime}, \quad \underline{\omega}(t) \leqq x \leqq \bar{\omega}(t) .
$$

Dans le cas du domaine non borné (7.2), il suffit, comme il l'a remarqué, de prendre pour $\Omega=\bar{\Omega}=-\underline{\Omega}$ la fonction d'une seule variable $x$ définie par

$$
\Omega \cdot d \Omega / d x=\varphi(\Omega), \quad \Omega(-M)=M,
$$

$M$ désignant un nombre positif tel que

$$
\left|\underline{\omega}^{+}(\alpha)\right|, \quad\left|\overline{\boldsymbol{\omega}}^{+}(\boldsymbol{\alpha})\right|<M .
$$


Remarque. Puisqu'on suppose $\underline{\omega}(\alpha)=\bar{\omega}(\alpha)$, et $\underline{\omega}(t)<\bar{\omega}(t)$ pour $\alpha<t \leqq \alpha^{\prime}$, on a

$$
\underline{\omega^{\prime}}(\alpha) \leqq \bar{\omega}^{\prime}(\alpha)
$$

\section{Rappel d'un théorème d'existence de H. Okamura.}

Rappelons encore un théorème d'existence dû à $H$. Okamura [1] . Les hypothèses relatives à $\omega$ et $\bar{\omega}$ sont les mêmes que dans le théorème de $M$. Nagumo sous la forme généralisée. Soit $\psi$ une fonction lipschitzienne telle que $\underline{\omega} \leqq \psi \leqq \bar{\omega}$ dans l'intervalle $\left[\alpha, \alpha^{\prime}\right]$. Supposons d'autre part l'existence des fonctions $\Phi_{1}, \Phi_{2}, \Psi_{1}, \Psi_{2}$ satisfaisant aux conditions suivantes:

Elles sont positives et continues respectivement dans les domaines fermés

$$
\begin{array}{lll}
\alpha \leqq t \leqq \alpha^{\prime}, & \omega(t) \leqq x \leqq \psi(t), & x^{\prime} \geqq K ; \\
\alpha \leqq t \leqq \alpha^{\prime}, & \psi(t) \leqq x \leqq \bar{\omega}(t), & x^{\prime} \leqq-K \\
\alpha \leqq t \leqq \alpha^{\prime}, & \psi(t) \leqq x \leqq \bar{\omega}(t), & x^{\prime} \geqq K \\
\alpha \leqq t \leqq \alpha^{\prime}, & \underline{\omega}(t) \leqq x \leqq \psi(t), & x^{\prime} \leqq-K
\end{array}
$$

et admettent les dérivées continues dans leurs intérieurs, où $K$ est une certaine constante positive. Elles convergent uniformément vers 0 lorsque $\left|x^{\prime}\right| \rightarrow \infty,\left(t, x, x^{\prime}\right)$ restant respectivement dans leurs domaines de définition et satisfont aux inégalités

$$
\begin{aligned}
& \frac{\partial \Phi_{i}}{\partial t}+\frac{\partial \Phi_{i}}{\partial x} x^{\prime}+\frac{\partial \Phi_{i}}{\partial x^{\prime}} f\left(t, x, x^{\prime}\right) \geqq 0, \quad i=1,2, \\
& \frac{\partial \Psi_{i}}{\partial t}+\frac{\partial \Psi_{i}}{x} x^{\prime}+\frac{\partial \Psi_{i}}{\partial x^{\prime}} f\left(t, x, x^{\prime}\right) \leqq 0, \quad i=1,2,
\end{aligned}
$$

dans les intérieurs de leurs domaines de définition.

Alors il existe au moins une solution prenant les valeurs $\psi(\alpha)$ et $\psi\left(\alpha^{\prime}\right)$ respectivement pour $t=\alpha$ et $t=\alpha^{\prime}$.

\section{Un théorème d'existence pour le système de deux équations différentielles.}

Nous avons établi antérieurement [5] un théorème d'exientsce 
pour le système de deux équations différentielles réelles

$$
d x / d t=f(t, x, y), \quad d y / d t=g(t, x, y) .
$$

En voici l'énoncé.

Hypothèse. $S_{1}(y, z)$ et $S_{2}(y, z)$ sont des fonctions continues convexes positives et semi-linéaires et $\omega_{1}(t)$ et $\omega_{2}(t)$ des fonctions positives et dérivables dans l'intervalle compact $\left[\alpha, \alpha^{\prime}\right\rceil$. Les fonctions $f$ et $g$ sont continues dans l'ensemble $\mathfrak{D}$ défini par

$$
\alpha \leqq t \leqq \alpha^{\prime}, \quad S(t, x, y) \leqq 1,
$$

où

$$
S(t, x, y)=\max \left\{S_{1}(x, y) / \omega_{1}(t), S_{2}(x, y) / \omega_{2}(t)\right\} .
$$

En désignant par $A_{i}, i=1,2$, les ensembles définis par

$$
\alpha \leqq t \leqq \alpha^{\prime}, \quad S_{i}(x, y) / \omega_{i}(t)=S(t, x, y)=1
$$

on a

$$
\omega_{i}^{\prime}(t)>S_{1}(f(t, x, y), \quad g(t, x, y))
$$

pour $(t, x, y) \in A_{1}$ et

$$
-\omega_{2}^{\prime}(t)>S_{2}(-f(t, x, y),-g(t, x, y))
$$

pour $(t, x, y) \in A_{2}$. On a enfin

$$
\frac{S_{1}(0, y)}{\omega_{1}(\alpha)}<\frac{S_{2}(0, y)}{\omega_{2}(\alpha)}, \quad \frac{S_{1}(x, 0)}{\omega_{1}\left(\alpha^{\prime}\right)}>\frac{S_{2}(x, 0)}{\omega_{2}\left(\alpha^{\prime}\right)} .
$$

Conclusion. Il existe au moins une solution telle que

$$
x(\alpha)=0, \quad y\left(\alpha^{\prime}\right)=0 .
$$

\section{Examen des conditions d'existence.}

On pourra obtenir de diverses conditions d'existence de plus en plus compliquées comme l'a remarqué $\mathrm{H}$. Okamura. Mais ne pourra-t-on pas espérer de les réunir en un théorème? Examinons la portée de nos théorèmes fondamentaux.

$1^{\circ}$ Considérons d'abord la condition d'existence du $\mathrm{n}^{\circ} 9 . \mathrm{La}$ 
frontière $\mathfrak{B}$ du domaine fondamental $\mathfrak{D}$ est l'ensemble des points $(t, x, y)$ tels que l'on ait ou bien

$$
t=\alpha, \quad S(\alpha, x, y) \leqq 1 ;
$$

ou bien

$(10.1)_{2}$

$$
t=\alpha^{\prime}, \quad S\left(\alpha^{\prime}, x, y\right) \leqq 1
$$

ou bien

$$
\alpha \leqq t \leqq \alpha^{\prime}, \quad S(t, x, y)=1 .
$$

Il est clair que le vacteur $(t, f(t, x, y), g(t, x, y))$ lié en $(t, x, y)$ se dirige vers l'extérieur de $\mathfrak{D}$ pour $(10.1)_{2}$ et vers l'intérieur de $\mathfrak{D}$ pour

$$
t=\alpha, \quad S(\alpha, x, y)<1 .
$$

Considérons maintenant un point $(\tau, \xi, \eta)$ où l'on a

$$
\alpha \leqq \tau<\alpha^{\prime}, \quad S(\tau, \xi, \eta)=1 .
$$

Si $x=x(t), y=y(t)$ représentent une courbe issue de $(\tau, \xi, \eta) \in A_{1} \cap A_{2}$ à droite, on a, en désignant par $D^{+}$la dérivation à droite par rapport à $t$,

$$
\begin{aligned}
D^{+} \frac{S_{1}(x(t), y(t))}{\omega_{1}(t)} & \leqq \frac{S_{1}\left(x^{\prime}(\tau), y^{\prime}(\tau)\right)-\omega_{1}^{\prime}(\tau)}{\omega_{1}(\tau)}<0, \\
D^{+} \frac{S_{2}(x(t), y(t))}{\omega_{2}(t)} & \geqq \frac{-S_{2}\left(-x^{\prime}(\tau),-y^{\prime}(\tau)\right)-\omega_{2}^{\prime}(\tau)}{\omega_{2}(\tau)}>0
\end{aligned}
$$

pour $t=\tau$ pourvu que

$$
\left|x^{\prime}(\tau)-f(\tau, \xi, \eta)\right|, \quad\left|y^{\prime}(\tau)-g(\tau, \xi, \eta)\right|
$$

soient assez petits. On en conclut que l'on a

$$
S(t, x(t), y(t))=\frac{S_{2}(x(t), y(t))}{\omega_{2}(t)}>\frac{S_{1}(x(t), y(t))}{\omega_{1}(t)}
$$

pour $t-\tau>0$ assez petit. On a évidemment ces relations si l'on a

$$
(\tau, \xi, \eta) \in A_{2}, \quad \notin A_{1},
$$


Par suite, si $(\tau, \xi, \eta) \in A_{2}$, on a, pour $t=\tau$,

$$
D^{+} S(t, x(t), y(t))=D^{+} \frac{S_{2}(x(t), y(t))}{\omega_{2}(t)}>0
$$

et $(t, x(t), y(t))$ se trouve à l'extérieur de $\mathscr{D}$ pour $t-\tau>0$ assez petit. Le vecteur $(1, f(\tau, \xi, \eta), g(\tau, \xi, \eta))$ lié en $(\tau, \xi, \eta)$ se dirige donc vers l'extérieur de $\mathfrak{D}$.

Si l'on a

$$
(\tau, \xi, \eta) \in A_{1}, \notin A_{2},
$$

on a l'égalité

$$
S(t, x(t), y(t))=\frac{S_{1}(x(t), y(t))}{\omega_{1}(t)}
$$

dans le voisinage de $\tau$, et en la dérivant, on obtient

$$
D^{+} S(t, x(t), y(t))<0,
$$

d'où l'on conclut que le vecteur $(1, f(\tau, \xi, \eta), g(\tau, \xi, \eta))$ lié en $(\tau, \xi, \eta)$ se dirige vers l'intérieur de $\mathfrak{D}$.

On peut faire un raisonnement analogue concernant le vecteur $-(1, f(\tau, \xi, \eta), g(\tau, \xi, \eta))$.

Cela posé, l'hypothèse II. 1 est évidemment remplie.

L'ensemble $\mathfrak{B}^{+}$des points frontières $(t, x, y)$ de $\mathfrak{D}$ où le vecteur $(1, f(t, x, y),, g(t, x, y))$ se dirige vers l'extérieur de $\mathfrak{D}$ se compose des points $(t, x, y)$ tels que l'on ait $(10.1)_{2}$ ou $(9.4)_{2}$ et l'ensemble $\mathfrak{B}^{-}$des points frontières $(t, x, y)$ où le vecteur $-(1, f(t, x, y)$, $g(t, x, y))$ se dirige vers l'extérieur de $\mathfrak{D}$ se compose des points $(t, x, y)$ tels que l'on ait $(10.1)_{1}$ ou (9.4) ${ }_{1}$. L'hypothèse II. 2 est donc remplie.

Les considérations ci-dessus montrent que l'hypothèse II. 3 est aussi remplie.

Prenons pour ç le segment défini par

$$
x(\alpha)=0, \quad S(\alpha, 0, y) \leqq 1
$$

et pour $\mathfrak{C}^{\prime}$ le segment défini par 


$$
y\left(\alpha^{\prime}\right)=0, \quad S\left(\alpha^{\prime}, x, 0\right) \leqq 1 .
$$

(5 est une partie continue de $\mathfrak{B}^{-}$et grâce à la première des inégalités (9.6), ses extrémités appartiennent à $\mathfrak{B}^{+}$.

$\mathbb{S}^{\prime}$ est une partie continue de $\mathfrak{B}^{+}$et grâce à la deuxième des inégalités (9.6), ses extrémités appartiennent à $\mathfrak{B}^{-}$.

Si $A=(\alpha, \beta)$ et $B=\left(\alpha, \beta^{\prime}\right)$ sont les extrémités de $\mathfrak{E}$, on a

$$
S_{2}(0, \beta)=\omega_{2}(\alpha), \quad S_{2}\left(0, \beta^{\prime}\right)=\omega_{2}(\alpha),
$$

et $\beta \beta^{\prime}<0$. Supposons par exemple $\beta<0, \beta^{\prime}>0$.

Soit $\mathbb{C}^{\prime \prime}$ une partie continue de $\mathfrak{B}^{+}$ne contenant aucun point de $\mathfrak{B}^{-}$sauf $A$ et $B$. Elle contient au moins un point $(\tau, \xi, 0)$ et si $\tau<\alpha^{\prime}$, on a

$$
\alpha<\tau<\alpha^{\prime}, \quad \frac{S_{1}(\xi, 0)}{\omega_{1}(\tau)} \leqq \frac{S_{2}(\xi, 0)}{\omega_{2}(\tau)} .
$$

Les inégalités $(9.5)_{1}$ et $(9.5)_{2}$ impliquent que $\omega_{1}$ et $-\omega_{2}$ sont croissantes. On a donc

$$
\frac{S_{1}(\xi, 0)}{\omega_{1}\left(\alpha^{\prime}\right)}<\frac{S_{2}(\xi, 0)}{\omega_{2}\left(\alpha^{\prime}\right)}
$$

contrairement à la deuxième des inégalités (9.6).

Si $\tau=\alpha^{\prime},(\tau, \xi, 0)$ appartient à $\varsigma^{\prime}$.

L'hypothèse II. 4 est donc remplie. Par conséquent le théorème cité au $\mathrm{n}^{\circ} 9$ peut être considéré comme un cas particulier du théorème d'existence 2 .

$2^{\circ}$ Considérons la condition d'existence de M. Nagumo. Nous supposerons les inégalités strictes (7.3) vérifiées et remplaçons l'équation différentielle (7.1) par le système différentiel équivalent

$$
d x / d t=y, \quad d y / d t=f(t, x, y) .
$$

Nous prenons pour le domaine fondamental $\mathfrak{D}$ l'ensemble défini par

$$
\alpha \leqq t \leqq \alpha^{\prime}, \quad \underline{\omega}(t) \leqq x \leqq \bar{\omega}(t), \quad \underline{\Omega}(t, x) \leqq y \leqq \bar{\Omega}(t, x) .
$$

Il n'est pas difficile de voir qu'il n'y a aucune caractéristique 
issue d'un point frontière $(t, x, y) \in \mathfrak{D}$ tel que l'on ait

$$
\alpha \leqq t \leqq \alpha^{\prime}, \quad x=\underline{\omega}(t), \quad y=\underline{\omega}^{\prime}(t)
$$

ou

$$
\alpha \leqq t \leqq \alpha^{\prime}, \quad x=\bar{\omega}(t), \quad y=\bar{\omega}^{\prime}(t) .
$$

Par suite la frontière gauche $\mathfrak{B}^{-}$de $\mathfrak{D}$ se compose de 5 parties:

$$
\begin{aligned}
& t=\alpha, \quad \underline{\omega}(t) \leqq x \leqq \bar{\omega}(t), \quad \underline{\Omega}(t, x) \leqq y \leqq \bar{\Omega}(t, x) ; \\
& \alpha \leqq t \leqq \alpha^{\prime}, \quad \underline{\omega}(t) \leqq x \leqq \bar{\omega}(t), \quad y=\underline{\Omega}(t, x) ; \\
& \alpha \leqq t \leqq \alpha^{\prime}, \quad \underline{\omega}(t) \leqq x \leqq \bar{\omega}(t), \quad y=\bar{\Omega}(t, x) ; \\
& \alpha \leqq t \leqq \alpha^{\prime}, \quad x=\underline{\omega}(t), \quad \underline{\omega}^{\prime}(t) \leqq y \leqq \bar{\Omega}(t, x) ; \\
& \alpha \leqq t \leqq \alpha^{\prime}, \quad x=\bar{\omega}(t), \quad \underline{\Omega}(t, x) \leqq y \leqq \bar{\omega}^{\prime}(t) ;
\end{aligned}
$$

et la frontière droite $\mathfrak{B}^{+}$de $\mathfrak{D}$ se compose de 3 parties

$$
t=\alpha^{\prime}, \quad \underline{\omega}(t) \leqq x \leqq \bar{\omega}(t), \quad \underline{\Omega}(t, x) \leqq y \leqq \bar{\Omega}(t, x) ;
$$

$$
\alpha \leqq t \leqq \alpha^{\prime}, \quad x=\underline{\omega}(t), \quad \underline{\Omega}(t, x) \leqq y \leqq \underline{\omega^{\prime}}(t) ;
$$

$$
\alpha \leqq t \leqq \alpha^{\prime}, \quad x=\bar{\omega}(t), \quad \bar{\omega}^{\prime}(t) \leqq y \leqq \bar{\Omega}(t, x) .
$$

Et en un point $(t, x, y)$ n'appartenant pas à l'intersection $\mathfrak{B}^{+} \cap \mathfrak{B}^{-}$l'un des vecteurs $\pm(1, y, f(t, x, y))$ se dirige vers l'intérieur de $\mathcal{D}$. Par suite les hypothèses I. $1 \sim 5$ sont remplies.

Soient $\mathfrak{C}$ et $\mathfrak{C}^{\prime}$ les segments définis respectivement par

$$
\begin{aligned}
& t=\alpha, \quad x=\beta, \\
& \underline{\omega}^{\prime}(\alpha) \leqq y \leqq \bar{\omega}^{\prime}(\alpha)
\end{aligned}
$$

et

$$
\begin{aligned}
& t=\alpha^{\prime}, \quad x=\beta^{\prime} \\
& \underline{\Omega}\left(\alpha^{\prime}, \beta^{\prime}\right) \leqq y \leqq \Omega\left(\alpha^{\prime}, \beta^{\prime}\right) . \\
& \mathfrak{S}^{\prime} \text { est contenu dans la }
\end{aligned}
$$
partie $(10.5)_{1}$, et l'intersection $\mathfrak{C}^{\prime} \cap \mathfrak{B}^{-}$se compose de deux

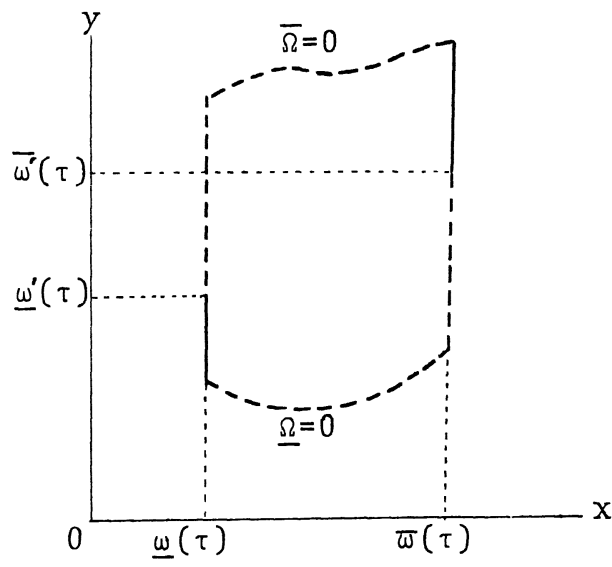

Fig. 1 
points

$$
\mathrm{A}=\left(\alpha^{\prime}, \beta^{\prime}, \underline{\Omega}\left(\alpha^{\prime}, \beta^{\prime}\right)\right) \quad \text { et } \mathrm{B}=\left(\alpha^{\prime}, \beta^{\prime}, \bar{\Omega}\left(\alpha^{\prime}, \beta^{\prime}\right)\right)
$$

et on ne peut joindre $A$ et $B$ par une partie continue de $\mathfrak{B}^{-}$sans recontrer $\mathfrak{E} \cup \mathfrak{B}^{+}$.

Le théorème d'existence 1 est donc applicable, intervertissant les rôles de $\sqrt{5}$ et $\mathbb{C}^{\prime}$.

Mais si l'on a $(7.3)^{\prime}$ au lieu de (7.3), le théorème 1 n'est plus applicable, puisqu'il y auraient des points latéraux sur la frontière de $\mathfrak{D}$.

$3^{0}$ Examinons enfin la condition d'existence de $\mathrm{H}$. Okamura. Comme dans le cas précédent, considérons le système différentiel (10.2) au lieu de (7.1) et supposons les inégalités strictes pour $\underline{\omega}, \overline{\boldsymbol{\omega}}$ et $\Phi_{i}, \Psi_{i}, i=1,2$. Le domaine fondamental $\mathfrak{D}$ est un ensemble limité par les hyperplans $t=\alpha, t=\alpha^{\prime}$ et l'intersection de $\mathfrak{D}$ et de l'hyperplan $t=\tau$ est l'ensemble limité par les quatre courbes

$$
\begin{array}{ll}
\underline{\omega}(\tau) \leqq x \leqq \psi(\tau), & \Phi_{1}(\tau, x, y)=\varepsilon, \\
\psi(\tau) \leqq x \leqq \bar{\omega}(\tau), & \Psi_{1}(\tau, x, y)=\varepsilon^{\prime} ; \\
\psi(\tau) \leqq x \leqq \bar{\omega}(\tau), & \Phi_{2}(\tau, x, y)=\varepsilon ; \\
\underline{\omega}(\tau) \leqq x \leqq \psi(\tau), & \Psi_{2}(\tau, x, y)=\varepsilon^{\prime} ;
\end{array}
$$

et les quatre segments qui les joignent bout à bout, comme le montrent les figures ci-dessous, $\varepsilon, \varepsilon^{\prime}$ désignant des nombres positifs assez petits.

Remarque. Si, après avoir défini $\varepsilon^{\prime}$, on prend $\varepsilon$ assez petit, le cas de la figure 2 aura lieu. Au contraire, si, après avoir défini $\varepsilon$, on prend $\varepsilon^{\prime}$ assez petit, le cas de la figure 3 aura lieu.

En un point $(\tau, x, y)$ situé sur $\mathrm{KAB}$ ou $\mathrm{LEF}$ le vecteur $(1, y$, $f(\tau, x, y)$ ) se dirige vers l'intérieur (K, B, L, F exclus) et le vecteur $-(1, y, f(\tau, x, y))$ se dirige vers l'extérieur (K, L exclus); au contraire en un point de CDL ou GHK le vecteur $-(1, y, f(\tau, x, y))$ se dirige vers l'intérieur ( $\mathrm{C}, \mathrm{L}, \mathrm{G}, \mathrm{K}$ exclus) et le vecteur $(1, y$, $f(\tau, x, y)$ ) se dirige vers l'extérieur ( $\mathrm{K}, \mathrm{L}$ exclus). On voit aussi comme dans le cas précédent de Nagumo, $K$ et $L$ appartiennent à 


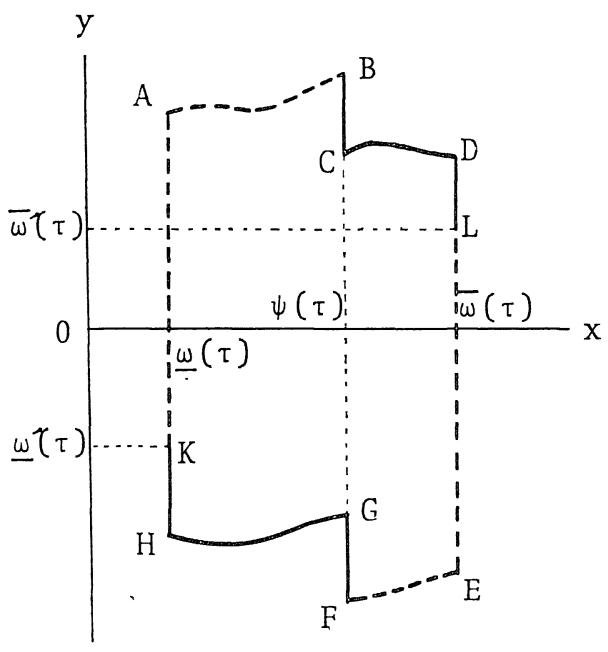

Fig. 2

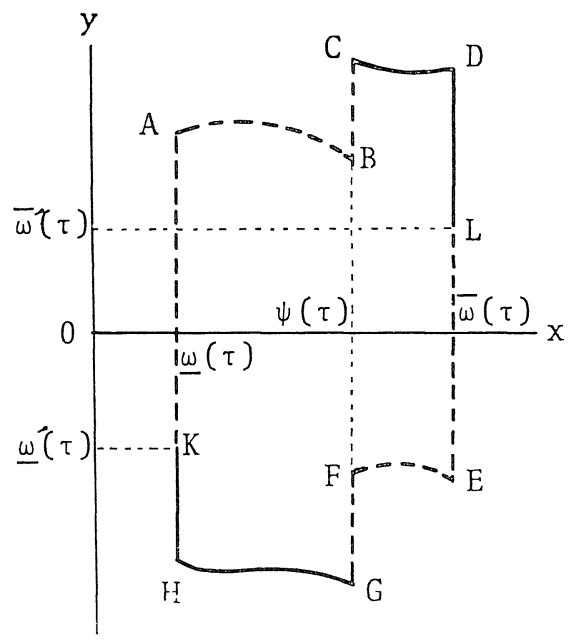

Fig. 3

l'intersection $\mathfrak{B}^{+} \cap \mathfrak{B}^{-}$.

Considérons maintenant un point $(\tau, x, y)$ du segment BC. Si B est au-dessus de $\mathrm{C}$, le vecteur $(1, y, f(\tau, x, y))$ se dirige vers l'extérieur (sans exception) et le vecteur $-(1, y, f(\tau, x, y))$ se dirige vers l'intérieur (B exclu) et $\mathrm{B}$ appartient à l'intersection $\mathfrak{B}^{+} \cap \mathfrak{B}^{-}$. Si B est au-dessous de $\mathrm{C}$, le vecteur $(1, y, f(\tau, x, y))$ se dirige vers l'intérieur (C exclu) et le vecteur - $(1, y, f(\tau, x, y))$ se dirige vers l'extérieur (sans exception) et $\mathrm{C}$ appartient à l'intersection $\mathfrak{B}^{+} \cap \mathfrak{B}^{-}$.

Il en est de même du segment FG.

Puisqu'on peut vérifier sans peine les autres propriétés exposées au $\mathrm{n}^{\circ} 1$, les hypothèses I. $1 \sim 5$ sont remplies.

Soient $\sqrt{5}$ le segment CG situé dans l'hyperplan $t=\alpha$ et $\Im^{\prime}$ le segment BF situé dans l'hyperplan $t=\alpha^{\prime}$. Les points $\mathrm{C}$ et $\mathrm{G}$ appartiennent à $\mathfrak{B}^{+}$. CDL et $\mathrm{GHK}$ sont toujours séparées par $\mathfrak{B}^{-}$pour $\alpha<\tau<\alpha^{\prime}$. Dans l'hyperplan $t=\alpha^{\prime}$ elles sont encore séparées par $\mathfrak{S}^{\prime} \cup \mathfrak{B}^{-}$. Le théorème d'existence 1 assure donc l'existence d'une caractéristique qui rencontre $\sqrt{5}$ et $\varsigma^{\prime}$. 


\section{BIBLIOGRAPHIE}

M. Hukuhara,

[1] Sur les systèmes des équations différentielles ordinaires. Proc. Imp. Acad., 4 (1928), 448-449.

[2] Sur l'ensemble des courbes intégrales d'un système d'équations différentielles ordinaires, I, II, III. Ibid., 6 (1930), 360-362; 7 (1931), 37-39; 298-299.

[3] Sur les familles de fonctions à une variable réelle. J. Fac. Sci., Hokkaido Imp. Univ., 1 (1932), 163-209.

[4] Sur les propriétés de la famille des courbes intégrales d'un système différentiel ordinaire. Proc. Japan Acad., 25 (1949), 151-153.

[5] Le problème aux limites pour un système de deux équations différentielles ordinaires. J. Math. Soc. Japan, 3 (1951), 99-103.

[6] Sur un théorème de Kneser. J. Fac. Sci., Univ. Tokyo, 6 (1953), 329-344.

[7] Sur une généralisation d'un théorème de Kneser. Proc. Japan Acad., 29 (1953), 154-155.

[8] Sur l'application qui fait correspondre à un point un continu bicompact. Ibid., 31 (1955), 5-7.

[9] Zyôbibunhôteisikiron (Théorie des équations différentielles ordinaires), Iwanami, Tokyo, 1933.

M. Hukuhara et M. Nagumo,

[1] Un théorème relatif à l'ensemble des courbes intégrales d'un système d'équations différentielles. Proc. Phys.-Math. Soc. Japan, 12 (1930), 233-239.

H. Kneser,

[1] Über die Lösungen eines Systems gewöhnlicher Differentialgleichungen, das der Lipschitzschen Bedingung nicht genügt. Sitzungsberichte Preusz. Akad. Wiss., Phys.-Math. K1., (1923). 171-174.

M. Nagumo,

[1] Über das System der gewöhnlichen Differentialgleichungen. Japanese J. Math., 4 (1927), 215-230.

[2] Über die Differentialgleichung $y^{\prime \prime}=f\left(x, y, y^{\prime}\right)$. Proc. Phys.-Math. Soc. Japan, 19 (1937), 861-866.

[3] Über das Randwertproblem der nicht linearen gewöhnlichen Differentialgleichungen zweiter Ordnung. Ibid., 24 (1942), 845-851.

[4] Eine Art der Randwertaufgabe von Systemen gewöhnlicher Differentialgleichungen, I, II. Ibid., 25 (1943), 221-226, 384-390.

[5] Kansû Kûkan ni okeru Syazô. Kansû Hôteisiki, No. 4 (1938), 10-22.

[6] Dai-2-kai Zyôbibunhôteisiki no Kyôkaiti Mondai, I, II. Ibid., No. 5 (1939), 27-34; No. 6 (1939), 37-44.

[7] $y^{\prime \prime}=f\left(x, y, y^{\prime}\right)$ no Kyôkaiti Mondai ni tuite, I, II. Ibid., No. 30 (1941), 36-46; No. 17 (1942), 50-52.

H. Okamura,

[1] $y^{\prime \prime}=f\left(x, y, y^{\prime}\right)$ ni tuite, I, II, III. Kansû Hôteisiki, No. 27 (1941), 27-35; No. 30 (1941), 14-19; No. 31 (1942) 32-40. 


\section{ERRATA}

Sur la figure 1 , on doit lire $y=\underline{\Omega}, y=\vec{\Omega}$ respectivement au lieu de $\underline{\Omega}=0, \bar{\Omega}=0$. 